\title{
NORMAL FAMILIES OF QUASIMEROMORPHIC MAPPINGS
}

\author{
RUTH MINIOWITZ ${ }^{1}$
}

\begin{abstract}
We obtain several sufficient conditions for a family of quasiregular or quasimeromorphic mappings to be normal, which are generalizations to known results for analytic functions.
\end{abstract}

1. Introduction. Zalcman in [Z] formulated a heuristic principle in Complex Function Theory that yielded simple proofs to some of Montel's theorems concerning normal families.

Here we generalize Zalcman's result to the Theory of Quasimeromorphic Mappings. We obtain several sufficient conditions for a family to be normal. We also obtain some $n$-dimensional versions of known theorems for analytic functions.

2. Preliminaries. We consider quasiregular and quasimeromorphic mappings $f: D \rightarrow \overline{\mathbf{R}^{n}}$ where $D$ is a domain in $\mathbf{R}^{n}$ or in $\overline{\mathbf{R}^{n}}$ and $n \geqslant 2$. We shall use the same notations and terminology as in [MRV1, MRV2, MRV3].

For the definitions and main properties of quasiregular and quasimeromorphic mappings, as well as related material, we refer the reader to those papers.

2.1. Normal families. A family $\mathscr{F}$ of $K$-quasimeromorphic mappings in a domain $D \subseteq \overline{\mathbf{R}^{n}}$ is called normal, if every sequence $\left\{f_{m}\right\} \subset \mathscr{F}$ has a subsequence that converges uniformly, in the spherical metric, on every compact subset contained in D.

The spherical distance $q(a, b)$ between two points $a, b \in \overline{\mathbf{R}^{n}}$ is defined by

$$
\begin{aligned}
q(a, b) & =|a-b|\left(1+|a|^{2}\right)^{-\frac{1}{2}} \cdot\left(1+|b|^{2}\right)^{-\frac{1}{2}}, \quad a, b \in \mathbf{R}^{n}, \\
q(a, \infty) & =\left(1+|a|^{2}\right)^{-\frac{1}{2}}
\end{aligned}
$$

\section{A necessary and sufficient condition for normality.}

THEOREM 1. Let $\mathcal{F}$ be a family of $K$-quasimeromorphic mappings in a domain $D$, $D \subseteq \overline{\mathbf{R}^{n}}, n>2$. Then $\mathcal{F}$ is a normal family if and only if for each compact subset $E$ of $\bar{D}$ there exists a finite number $M$ such that

$$
q\left(f\left(x_{1}\right), f\left(x_{2}\right)\right)<M\left(q\left(x_{1}, x_{2}\right)\right)^{\alpha}
$$

for each $x_{1} \in E, x_{2} \in D$ and $f \in \mathcal{F}$ where $\alpha=(K)^{1 /(1-n)}$.

Received by the editors December 12, 1980 and, in revised form, March 5, 1981.

1980 Mathematics Subject Classification. Primary 30D45.

${ }^{1}$ The author wishes to thank Professor D. M. Campbell, Professor F. W. Gehring, Professor U. Srebro and Professor L. Zalcman for their useful remarks. 
Proof. The sufficiency is clear, since it implies equicontinuity at each point, and by Ascoli's theorem, equicontinuity implies normality. For the necessary part, suppose $\mathscr{F}$ is a normal family in $D$, and $x_{0}$ be any arbitrary point in $D$. As $\mathscr{F}$ is normal it is equicontinuous at $x_{0}$, and consequently there exists a positive number $\delta<1$, such that $q\left(x, x_{0}\right)<\delta$ is contained in $D$ and $q\left(f(x), f\left(x_{0}\right)\right)<1$ whenever $q\left(x, x_{0}\right)<\delta$ and $f \in \mathscr{F}$. Let $T$ be a Möbius transformation that corresponds to a rotation of the Riemann sphere, that maps $x_{0}$ to the origin, and denote by $T^{-1}$ its inverse mapping. $T$ maps the neighborhood $q\left(x, x_{0}\right)<\delta$, onto a ball $B^{n}(0, r)$, $r<1$. For each $f \in \mathscr{F}$, we denote by $U$ a Möbius transformation that corresponds to a rotation of the Riemann sphere that takes $f\left(x_{0}\right)$ onto the origin. The mappings $v=U \circ f \circ T^{-1}$ are $K$-quasiregular in $T(D)$. As $U$ corresponds to a rotation of the Riemann sphere, we have $|v(\zeta)|<1$ whenever $|\zeta|<r$. By [MRV2, 3.4] we can get that if $\left|\zeta_{1}\right|<r / 2,\left|\zeta_{2}\right|<r / 2$,

$$
\left|v\left(\zeta_{1}\right)-v\left(\zeta_{2}\right)\right|<\left(2 \lambda_{n} /\left(r-\frac{r}{2}\right)^{\alpha}\right)\left|\zeta_{1}-\zeta_{2}\right|^{\alpha}
$$

where $\lambda_{n}$ is a constant that depends only on $n, \lambda_{n}>1$, and $\alpha=(K)^{1 /(1-n)}$. Thus

$$
\left|v\left(\zeta_{1}\right)-v\left(\zeta_{2}\right)\right| \leqslant M_{0}\left|\zeta_{1}-\zeta_{2}\right|^{\alpha}
$$

where $M_{0}=2^{\alpha+1} \lambda_{n} / r^{\alpha}$ depends on $x_{0}, n$ and $K$. In the ball $|\zeta|<r$ we have

$$
d q(\zeta)=\frac{|d \zeta|}{\left(1+|\zeta|^{2}\right)} \geqslant \frac{d|\zeta|}{\left(1+r^{2}\right)}
$$

thus

$$
q\left(\zeta_{1}, \zeta_{2}\right) \geqslant \frac{\left|\zeta_{1}-\zeta_{2}\right|}{\left(1+r^{2}\right)}
$$

Therefore for each $\left|\zeta_{1}\right|<r / 2,\left|\zeta_{2}\right|<r / 2$

$$
\left|v\left(\zeta_{1}\right)-v\left(\zeta_{2}\right)\right| \leqslant M_{0}\left(1+r^{2}\right)^{\alpha}\left(q\left(\zeta_{1}, \zeta_{2}\right)\right)^{\alpha} \text {, }
$$

or

$$
\begin{aligned}
q\left(f\left(x_{1}\right), f\left(x_{2}\right)\right) & =q\left(v\left(\zeta_{1}\right), v\left(\zeta_{2}\right)\right) \leqslant\left|v\left(\zeta_{1}\right)-v\left(\zeta_{2}\right)\right| \\
& \leqslant M_{0}^{\prime}\left(q\left(\zeta_{1}, \zeta_{2}\right)\right)^{\alpha}=M_{0}^{\prime}\left(q\left(x_{1}, x_{2}\right)\right)^{\alpha}
\end{aligned}
$$

for each $f \in \mathcal{F}$ and each $x_{1}, x_{2}$ in $q\left(x, x_{0}\right)<\delta^{\prime}$.

Let $E$ be any compact subset of $D$, for each $p \in E$ there exist constants $k_{p}$ and $M_{p}$ such that $q\left(f\left(x_{1}\right), f\left(x_{2}\right)\right) \leqslant M_{p}\left(q\left(x_{1}, x_{2}\right)\right)^{\alpha}$ whenever $q\left(x_{1}, p\right)<k_{p}, q\left(x_{2}, p\right)<k_{p}$ and $f \in \mathscr{F}$. The rest of the proof is the same as [V1, Theorem 1].

REMARK. For plane quasimeromorphic mappings Theorem 1 is known, see [V1, Theorem 1].

\section{The main lemma.}

LEMMA 1. A family $\mathscr{F}$ of $K$-quasimeromorphic mappings in the unit ball $B^{n}$ is not normal if and only if there exist

(a) a number $0<r<1$,

(b) points $x_{m}, x_{m}$ in $B^{n}(r)$, 
(c) mappings $f_{m} \in \mathcal{F}$,

(d) numbers $\rho_{m} \rightarrow 0^{+}$,

such that

$$
f_{m}\left(x_{m}+\rho_{m} \zeta\right) \rightarrow g(\zeta)
$$

spherically uniformly on compact subsets of $\mathbf{R}^{n}$, where $g$ is a nonconstant quasimeromorphic mapping.

Proof. Suppose $\mathcal{F}$ is not normal on $B^{n}$, then it is not normal on some compact subset that is contained in $B^{n}\left(r^{*}\right), r^{*}<1$. By Theorem 1 there exist two sequences of points $x_{m}^{*}$ and $y_{m}^{*}$ in $B^{n}\left(r^{*}\right)$ and a sequence $f_{m} \in \mathscr{F}$ such that

$$
\lim _{m \rightarrow \infty} \frac{q\left(f_{m}\left(x_{m}^{*}\right), f_{m}\left(y_{m}^{*}\right)\right)}{q\left(x_{m}^{*}, y_{m}^{*}\right)^{\alpha}}=+\infty, \quad \alpha=(K)^{1 /(n-1)} .
$$

It should be noted that $\alpha<1$.

Fix $r, r^{*}<r<1$ and let

$$
M_{m}=\sup _{\substack{|x|<r \\|y|<r}}\left(1-\frac{|x|^{2}}{r^{2}}\right) \frac{q\left(f_{m}(x), f_{m}(y)\right)}{q(x, y)^{\alpha}}, \quad \alpha=(K)^{1 /(1-n)} .
$$

It is clear that $M_{m} \rightarrow \infty$.

Let $x_{m}$ and $y_{m}$ be sequences such that

$$
\frac{q\left(f_{m}\left(x_{m}\right), f_{m}\left(y_{m}\right)\right)}{q\left(x_{m}, y_{m}\right)^{\alpha}}\left(1-\frac{\left|x_{m}\right|^{2}}{r^{2}}\right) \geqslant \frac{M_{m}}{2},
$$

and let $g_{m}(\zeta)=f_{m}\left(x_{m}+\rho_{m} \zeta\right)$, where

$$
\rho_{m}^{\alpha}=\frac{q\left(x_{m}, y_{m}\right)^{\alpha}}{q\left(f_{m}\left(x_{m}\right), f_{m}\left(y_{m}\right)\right)} .
$$

As $\rho_{m}^{\alpha}<\left(2 / M_{m}\right)\left(1-\left|x_{m}\right|^{2} / r^{2}\right)$ it follows that $\rho_{m} \rightarrow 0$ as $m \rightarrow \infty$. The mappings $g_{m}(\zeta)$ are defined for $|\zeta|<R_{m}$ where $R_{m}=\left(r-\left|x_{m}\right|\right) / \rho_{m}$. As

$$
\frac{1}{R_{m}}=\frac{\rho_{m}}{r-\left|x_{m}\right|} \leqslant \frac{2\left(r+\left|x_{m}\right|\right) \rho_{m}^{1-\alpha}}{r^{2} M_{m}} \leqslant \frac{4 \rho_{m}^{1-\alpha}}{r M_{m}}
$$

and $\alpha<1$, it follows that $R_{m} \rightarrow \infty$ as $m \rightarrow \infty$.

We shall show that $\left\{g_{m}\right\}$ is a normal family. Take $\zeta_{1}, \zeta_{2}$ such that $\left|\zeta_{1}\right|,\left|\zeta_{2}\right|<R$ and choose $R<R_{m}$ and $\left|x_{m}+\rho_{m} \zeta_{i}\right|<r, i=1,2$. Then using the definition of $M_{m}$ we get

$$
\begin{aligned}
\frac{q\left(g_{m}\left(\zeta_{1}\right), g_{m}\left(\zeta_{2}\right)\right)}{q\left(\zeta_{1}, \zeta_{2}\right)^{\alpha}} & \leqslant \frac{\rho_{m}^{\alpha}\left(1+R^{2}\right)^{\alpha} q\left(f_{m}\left(x_{m}+\rho_{m} \zeta_{1}\right), f_{m}\left(x_{m}+\rho_{m} \zeta_{2}\right)\right)}{\left[\frac{\left|\left(x_{m}+\rho_{m} \zeta_{1}\right)-\left(x_{m}+\rho_{m} \zeta_{2}\right)\right|}{\left(1+\left|x_{m}+\rho_{m} \zeta_{1}\right|^{2}\right)^{\frac{1}{2}}\left(1+\left|x_{m}+\rho_{m} \zeta_{2}\right|^{2}\right)^{\frac{1}{2}}}\right]^{\alpha}} \\
& \leqslant \frac{\rho_{m}^{\alpha}\left(1+R^{2}\right)^{\alpha} M_{m}}{\left(1-\frac{\left|x_{m}+\rho_{m} \zeta_{1}\right|^{2}}{r^{2}}\right)} .
\end{aligned}
$$


But as $\rho_{m}^{\alpha}<\left(2 / M_{m}\right)\left(1-\left|x_{m}\right|^{2} / r^{2}\right)$ it follows that

$$
\frac{q\left(g_{m}\left(\zeta_{1}\right), g_{m}\left(\zeta_{2}\right)\right)}{q\left(\zeta_{1}, \zeta_{2}\right)^{\alpha}}<\frac{\left(r^{2}-\left|x_{m}\right|^{2}\right)}{\left(r^{2}-\left|x_{m}+\rho_{m} \zeta_{1}\right|^{2}\right)} \cdot 2\left(1+R^{2}\right)^{\alpha}
$$

Thus

$$
\frac{q\left(g_{m}\left(\zeta_{1}\right), g_{m}\left(\zeta_{2}\right)\right)}{q\left(\zeta_{1}, \zeta_{2}\right)^{\alpha}}<\frac{r+\left|x_{m}\right|}{r+\left|x_{m}\right|+R \rho_{m}} \cdot \frac{r-\left|x_{m}\right|}{r-\left|x_{m}\right|-R \rho_{m}} \cdot 2\left(1+R^{2}\right)^{\alpha}
$$

The first factor is bounded by 1 ; the second factor for fixed $R$ tends to 1 as $m$ tends to $\infty$. Thus by Theorem $1,\left\{g_{m}\right\}$ is a normal family. Taking a subsequence, if necessary, we can assume that $g_{m}$ converges uniformly on compact subsets of $\mathbf{R}^{n}$ to a quasimeromorphic mapping $g$ or a constant. We want to show that $g$ is not a constant. Take a sequence $\zeta_{m}$ such that $x_{m}+\rho_{m} \zeta_{m}=y_{m}$ (it is possible as $\left|y_{m}\right|<r$ ). It is easy to check that $\left|\zeta_{m}\right|^{\alpha} \leqslant 2\left(1+r^{2}\right)^{\alpha}$ and therefore $\left\{\zeta_{m}\right\}$ contains a convergent subsequence. Thus for that subsequence

$$
\begin{aligned}
\frac{q\left(g_{m}(0), g_{m}\left(\zeta_{m}\right)\right)}{q\left(0, \zeta_{m}\right)^{\alpha}} & =\frac{\rho_{m}^{\alpha} q\left(f_{m}\left(x_{m}\right), f_{m}\left(y_{m}\right)\right)}{q\left(x_{m}, y_{m}\right)^{\alpha}\left(1+\left|x_{m}\right|^{2}\right)^{\alpha / 2}} \cdot \frac{\left(1+\left|\zeta_{m}\right|^{2}\right)^{\alpha / 2}}{\left(1+\left|y_{m}\right|^{2}\right)^{\alpha / 2}} \\
& =\frac{\left(1+\left|\zeta_{m}\right|^{2}\right)^{\alpha / 2}}{\left(1+\left|x_{m}\right|^{2}\right)^{\alpha / 2}\left(1+\left|y_{m}\right|^{2}\right)^{\alpha / 2}}>\frac{\left(1+\left|\zeta_{m}\right|^{2}\right)^{\alpha / 2}}{\left(1+r^{2}\right)^{\alpha}}
\end{aligned}
$$

Thus

$$
\frac{q\left(g_{m}(0), g_{m}\left(\zeta_{m}\right)\right)}{q\left(0, \zeta_{m}\right)^{\alpha}}>\frac{1}{\left(1+r^{2}\right)^{\alpha}}
$$

As $g_{m}$ is a normal family it is equicontinuous at $x=0$; then we follow the same argument as in the proof of Theorem 1 . We can find a positive number $a$ such that $\left|\zeta_{m}\right|<a$ and $g_{m}\left(B^{n}(a)\right)$ is defined for $m$ large enough. Then we define a sequence of Möbius transformations $U_{m}$ that correspond to rotations of the Riemann sphere that takes $g_{m}(0)$ onto the origin. The sequence $h_{m}=U_{m} \circ g_{m}$ forms a normal family of $K$-quasiregular bounded mappings on $B^{n}(a)$ of Theorem 1. By the definition of $U_{m}$ and [MRV2, 3.4] we get

$$
\begin{aligned}
\frac{q\left(g_{m}(0), g_{m}\left(\zeta_{m}\right)\right)}{q\left(0, \zeta_{m}\right)^{\alpha}} & =\frac{q\left(h_{m}(0), h_{m}\left(\zeta_{m}\right)\right)}{q\left(0, \zeta_{m}\right)^{\alpha}} \\
& =\frac{\left|h_{m}\left(\zeta_{m}\right)\right|}{\left(1+\left|h_{m}\left(\zeta_{m}\right)\right|^{2}\right)^{\frac{1}{2}}} \cdot \frac{1}{q\left(0, \zeta_{m}\right)^{\alpha}} \leqslant \frac{\lambda_{n}\left(1+a^{2}\right)^{\alpha / 2}}{a^{\alpha}} d\left(h_{m}\left(B^{n}(a)\right)\right)
\end{aligned}
$$

where $\lambda_{n}$ is a positive constant that depends on $n$, and $d(A)$ is the diameter of a set $A$. Thus by (*)' it follows that

$$
\frac{1}{\left(1+r^{2}\right)^{\alpha}} \leqslant \frac{\lambda_{n}\left(1+a^{2}\right)^{\alpha / 2}}{a^{\alpha}} d\left(h_{m}\left(B^{n}(a)\right)\right) .
$$


If $g_{m}$ converges to $g$ and $g \equiv$ constant then we can find a subsequence $m_{j}$ such that $h_{m}$ converges to $h$ and $h \equiv$ constant. But if $\lim _{m \rightarrow \infty} d\left(h_{m}\left(B^{n}(a)\right)\right)>\delta>0, h \neq$ constant; therefore, $g$ constant.

For the converse, suppose that $\mathscr{F}$ is normal in $B^{n}$. By Theorem 1 there exists a constant $M>0$ such that

$$
\sup _{\substack{|x|<(1+r) / 2 \\|y|<(1+r) / 2}} \frac{q(f(x), f(y))}{q(x, y)^{\alpha}}<M
$$

for all $f \in \mathscr{F}$.

Suppose (*) holds, fix $\zeta_{1}, \zeta_{2} \in \mathbf{R}^{n}$, then for large $m$ and $\left|x_{m}+\rho_{m} \zeta_{i}\right|<(1+r) / 2$, $i=1,2$, we get

$$
\frac{\rho_{m}^{\alpha} q\left(f_{m}\left(x_{m}+\rho_{m} \zeta_{1}\right), f_{m}\left(x_{m}+\rho_{m} \zeta_{2}\right)\right)}{q\left(x_{m}+\rho_{m} \zeta_{1}, x_{m}+\rho_{m} \zeta_{2}\right)^{\alpha}}<\rho_{m}^{\alpha} M
$$

or equivalently,

$$
\frac{q\left(g_{m}\left(\zeta_{1}\right), g_{m}\left(\zeta_{2}\right)\right)}{\left|\zeta_{1}-\zeta_{2}\right|^{\alpha}}\left(1+\left|x_{m}+\rho_{m} \zeta_{1}\right|^{2}\right)^{\alpha / 2}\left(1+\left|x_{m}+\rho_{m} \zeta_{2}\right|^{2}\right)^{\alpha / 2}<\rho_{m}^{\alpha} M
$$

Thus

$$
\frac{q\left(g_{m}\left(\zeta_{1}\right), g_{m}\left(\zeta_{2}\right)\right)}{\left|\zeta_{1}-\zeta_{2}\right|^{\alpha}} \leqslant \rho_{m}^{\alpha} M
$$

Therefore

$$
\frac{q\left(g\left(\zeta_{1}\right), g\left(\zeta_{2}\right)\right)}{\left|\zeta_{1}-\zeta_{2}\right|^{\alpha}}=\lim _{m \rightarrow \infty} \frac{q\left(g_{m}\left(\zeta_{1}\right), g_{m}\left(\zeta_{2}\right)\right)}{\left|\zeta_{1}-\zeta_{2}\right|^{\alpha}}=0 .
$$

But as $\zeta_{1}$ and $\zeta_{2}$ are any two points in $\mathbf{R}^{n}$, it follows that $g$ is a constant (possibly $\infty)$.

5. The heuristic principle. Following the notation of $[Z]$, we write $\{f, D\}$ to denote that the mapping $f$ is defined on the domain $D \subset \mathbf{R}^{n}$, and we distinguish between mappings $\{f, D\}$ and $\left\{f, D^{\prime}\right\}$ if $D \neq D^{\prime}$. For meromorphic functions the following theorem was proved by Zalcman in $[\mathrm{Z}]$; the same proof holds for quasimeromorphic mappings in $\mathbf{R}^{n}, n>2$.

THEOReM 2. Let $P$ be a property (that is a family of $K$-quasiregular or $K$-quasimeromorphic mappings) such that

(i) If $\{f, D\} \in P$ and $D^{\prime} \subset D$ then $\left\{f, D^{\prime}\right\} \in P$.

(ii) If $\{f, D\} \in P$ and $\phi(x)=a T(x)+b, a \in \mathbf{R}^{+}, T$ is an orthogonal mapping and $b \in \mathbf{R}^{n}$, then $\left\{f \circ \phi, \phi^{-1}(D)\right\} \in P$.

(iii) If $\left\{f_{m}, D_{m}\right\} \in P$ where $D_{1} \subset D_{2} \subset \cdots \subset D_{m} \subset \ldots$, and $D=\cup_{m=1}^{\infty} D_{m}$, $f_{m} \rightarrow f$ uniformly on compact subsets of $D$, then $\{f, D\} \in P$.

If $\left\{f, \mathbf{R}^{n}\right\} \in P$ implies that $f$ is a constant mapping, then for every domain $D \subset \mathbf{R}^{n}$ the family of mappings $\mathscr{F}=\{f:\{f, D\} \in P\}$ is normal on $D$. 
6. Applications. The ability to use Theorem 2 depends on finding conditions that will force a quasiregular mapping in $\mathbf{R}^{n}$ to be a constant mapping. The following theorem of Rickman's, an n-dimensional version of Picard's Little Theorem, is one such condition.

THEOREM 3 (RICKMAN [R]). For every integer $n \geqslant 2$ and each $K \geqslant 1$ there exists a positive integer $l=l(n, K)$ such that if $f: \mathbf{R}^{n} \rightarrow \mathbf{R}^{n} \backslash\left\{a_{1}, a_{2}, \ldots, a_{l}\right\}$ is $K$-quasiregular and $a_{1}, \ldots, a_{l}$ are distinct points in $\mathbf{R}^{n}$, then $f$ is a constant mapping.

We also need the following lemma, which is an $n$-dimensional version of Hurwitz's Theorem.

LEMMA 2. Let $f_{m}: D \rightarrow \mathbf{R}^{n} \backslash\{a\}$ be a sequence of $K$-quasiregular mappings that converge uniformly on every compact subset of $D$ to a $K$-quasiregular mapping $f$. Then $f$ is either a constant mapping or $n(a, f, D)=0(n(a, f, D)$ is the multiplicity function).

Proof. Suppose that $f$ is not constant and that there exists a point $x_{0} \in D$ such that $f\left(x_{0}\right)=a$. Let $r$ be a positive number such that $U=U\left(x_{0}, f, r\right)$, the $x_{0}$ component of $f^{-1}\left(B^{n}\left(f\left(x_{0}\right), r\right)\right)$ is a normal neighborhood of $x_{0}$. Such a $U$ exists by [MRV1, 2.10]. If we choose $r$ sufficiently small, then for $m$ sufficiently large, the mappings $f_{m}$ are defined on $\overline{U\left(x_{0}, f, r\right)}$ and converge uniformly to $f$. Denote $\partial U\left(x_{0}, f, r\right)$ by $R$, then $R$ is a compact set and as $\lim _{m \rightarrow \infty} f_{m}=f, f_{m}(R) \rightarrow$ $S^{n-1}\left(f\left(x_{0}\right), r\right)$ uniformly. Let $U_{m}$ be the component of $\mathbf{R}^{n} \backslash f_{m}(R)$ that contains $f\left(x_{0}\right)$, then $\mu\left(y, f_{m}, U\right)$ (the topological index) is constant for every $y \in U_{m}$, but this is possible only if $\mu\left(y, f_{m}, U\right)=0$ as $n\left(f\left(x_{0}\right), f_{m}, D\right)=0$. But $f_{m}(R)$ converging to $S^{n-1}\left(f\left(x_{0}\right), r\right)$, and $f_{m}\left(x_{0}\right)$ converging to $f\left(x_{0}\right)$ implies that for sufficiently large $m$, $\mu\left(y, f_{m}, U\right)>0$ for $y \in U_{m}$. This is a contradiction to $\mu\left(y, f_{m}, U\right)=0$. Thus if $f$ is not constant then $n(a, f, D)=0$.

THEOREM 4. Let $\mathcal{F}$ be a family of $K$-quasimeromorphic mappings in a domain $D$, $D \subseteq \mathbf{R}^{n}, n \geqslant 2$. Let $l=l(n, K)$ be the number guaranteed in Theorem 3. If there exists a set of different points in $\overline{\mathbf{R}^{n}}, A=\left\{a_{1}, \ldots, a_{l+1}\right\}$ such that $f(D) \cap A=\varnothing$ for all $f \in \mathcal{F}$, then $\mathcal{F}$ is a normal family.

Proof. Let $P$ be the property "either $f$ is constant or $f$ omits the points $a_{1}, \ldots, a_{l+1}$." It is obvious that conditions (i) and (ii) of Theorem 2 are satisfied. In order that (iii) be satisfied, we can assume that one of the omitted points is $\infty$ (otherwise let $h$ be a Möbius transformation that maps one point to $\infty$ and we consider the family $\{h \circ f\}$ where $f \in \mathscr{F}$ ). (iii) will follow immediately from Lemma 2. Theorem 3 guarantees that if $\left\{f, \mathbf{R}^{n}\right\} \in P$ then $f$ is a constant mapping. Thus we obtain that $\mathscr{F}$ is a normal family.

REMARKs. (i) For families of $K$-quasiregular mappings the point $\infty$ is always omitted so we need only to require that $l(n, K)$ points are omitted in Theorem 4.

(ii) Theorem 4 for analytic functions is known as Montel's Theorem. 
THEOREM 5. Let $\mathcal{F}$ be a family of $K$-quasimeromorphic mappings in a domain $D$, $D \subseteq \mathbf{R}^{n}, n \geqslant 2$. Let $l=l(n, K)$ be the number in Theorem 3. Suppose there exists $a$ positive number $\varepsilon$, such that

(1) each $f \in \mathscr{F}$ omits $l+1$ points $a_{1}(f), \ldots, a_{l+1}(f)$,

(2) $q\left(a_{i}(f), a_{j}(f)\right) \geqslant \varepsilon>0$ when $i \neq j, 1 \leqslant i, j \leqslant l+1$.

Then $\mathcal{F}$ is a normal family.

Proof. The proof will be based again on Theorem 2 .

Let $P$ be the property: " $f$ omits $l+1$ distinct points $a_{1}(f), \ldots, a_{l+1}(f)$ such that

$$
q\left(a_{i}(f), a_{j}(f)\right) \geqslant \varepsilon>0, \quad i \neq j, \quad 1 \leqslant i, j \leqslant l+1,
$$

or $f$ is a constant." The rest of the proof follows verbatim the proof of the Extended Montel Theorem in [Z].

We now present an $n$-dimensional version for Picard's Big Theorem. The proof is similar to the one in [SZ, pp. 351-352]. Another $n$-dimensional version for this theorem can be found in [R]; one can obtain Theorem 6 by the same methods that are in $[\mathbf{R}]$.

THEOREM 6. Let $f: B^{n} \backslash\{0\} \rightarrow \mathbf{R}^{n}$ be a $K$-quasiregular mapping and $x=0$ be an essential singularity for $f$. Let $l=l(n, K)$ be the number in Theorem 3. Then for all but at most $l-1$ exceptional values of $y$ in $\mathbf{R}^{n}, f$ takes any $y$ infinitely often in $B^{n} \backslash\{0\}$.

Proof. Let us assume that there exists $l$ distinct points $a_{1}, \ldots, a_{l} \in \mathbf{R}^{n}$ which $f$ assumes only finitely often in $B^{n} \backslash\{0\}$. Let $G$ be the spherical ring $B^{n} \backslash \overline{B^{n}(1 / 2)}$, and consider in $G$ the sequence $f_{j}(x)=f\left(\left(1 / 2^{j-1}\right) x\right)$. The mappings $f_{j}$ map $G$ onto the same domain that the mapping $f$ maps the spherical ring $B^{n}\left(1 / 2^{j-1}\right) \backslash B^{n}\left(1 / 2^{j}\right)$.

For $j$ sufficiently large each $f_{j}$ maps $G$ to $\mathbf{R}^{n} \backslash\left\{a_{1}, \ldots, a_{l}\right\}$ as $n\left(a_{k}, f, B^{n} \backslash\{0\}\right)$ $<\infty$ for $1 \leqslant k \leqslant l$. By Theorem 4 the family $\left\{f_{j}\right\}$ is a normal family in $G$, thus we can find a subsequence that on any sphere $S^{n-1}(r), \frac{1}{2}<r<1$, which is a compact subset of $G$, is either bounded or tends uniformly to $\infty$, but by [MRV2, 4.2] that means that $x=0$ is not an essential singularity, which is a contradiction.

Similarly one can get also an $n$-dimensional version of Julia's Theorem that can be formulated as follows.

THEOREM 7. Let $f: B^{n} \backslash\{0\} \rightarrow \mathbf{R}^{n}$ be a $K$-quasiregular mapping with an essential singularity at $x=0$, and let $l=l(n, K)$ be the number in Theorem 3. Then there exists a point $x_{0}$ in $D$ such that for each $\varepsilon>0, n(y, f, R)=\infty$ with at most $l-1$ exceptions for $y$, where

$$
R=\bigcup_{j} R_{j} ; \quad R_{j}=B^{n}\left(\frac{1}{2^{j}} x_{0}, \frac{\varepsilon}{2^{j}}\right) .
$$

Using Theorem 2 one can get also some known sufficient conditions for normality for families of $K$-quasimeromorphic local homeomorphisms, quasiconformal, and conformal mappings. We shall point out some of them. We need the following lemma. 
LEMMA 3. Let $f_{m}: D_{m} \rightarrow \mathbf{R}^{n}$ be a sequence of $K$-quasiregular mappings that are local homeomorphisms and $D_{1} \subset D_{2} \subset \cdots \subset D_{m} \subset \ldots$ If $D=\cup_{m=1}^{\infty} D_{m}, f_{m} \rightarrow f$ uniformly on compact subsets of $D$, then $f$ is a $K$-quasiregular mapping that is a local homeomorphism or $f$ is a constant.

PROof. If $D_{m}=B^{n}$ for every $m$ then it follows from [Sa, Lemma 4.2]. For any sequence of domain, take $x_{0} \in D$, then for $m \geqslant m_{0}, x_{0} \in D_{m}$. By [MRV3, 2.7] we can conclude that there exists a positive number $r=r(n, K)$ such that any $f_{m}$ (may be except a finite number) is $K$-quasiconformal in the ball $B^{n}\left(x_{0}, r \cdot d\right)$ where $d=\operatorname{dist}\left\{x_{0}, \partial D\right\} / 2$. As the limit of a sequence of $K$-quasiconformal mappings that converges uniformly on compact subsets is $K$-quasiconformal or a constant, we can conclude that the limit function is $K$-quasiconformal or a constant in $B^{n}\left(x_{0}, r \cdot d\right)$. As $x_{0}$ was an arbitrary point it follows that $f$ is either a constant mapping or a $K$-quasiregular mapping that is a local homeomorphism.

THEOREM 8 [MRV3, 2.9]. Let $D$ be a domain in $\mathbf{R}^{n}, n>3, K>1$, and $\varepsilon>0$. If $\mathscr{F}=\{f\}$ is a family of $K$-quasimeromorphic local homeomorphisms $f: D \rightarrow \overline{\mathbf{R}^{n}}$ such that every $f \in \mathcal{F}$ omits two points $a_{f}, b_{f} \in \overline{\mathbf{R}^{n}}$ with $q\left(a_{f}, b_{f}\right)>\varepsilon>0$, then $\mathscr{F}$ is $a$ normal family.

Proof. We can assume that one of the points $a_{f}$ or $b_{f}$ is $\infty$; otherwise, we can compose each mapping with a Möbius transformation that will correspond to a rotation of the Riemann sphere and that takes one of the points to $\infty$. Then we can talk about the new family.

Let $P$ be the property: " $f$ is a $K$-quasimeromorphic local homeomorphism that omits two points $a_{f}, \infty$ such that $q\left(a_{f}, \infty\right) \geqslant \varepsilon>0$ or $f$ is a constant." Conditions (i) and (ii) of Theorem 2 are clearly satisfied; condition (iii) is fulfilled by Lemma 3. By [Zo] any $K$-quasiregular local homeomorphism of $\mathbf{R}^{n}$ is a global homeomorphism. But if $f$ is $K$-quasiconformal then as of topological reasons it follows that if $\left\{f, \mathbf{R}^{n}\right\} \in P$ then $f$ is a constant mapping. Thus $f$ is a normal family of Theorem 2 .

As a corollary we can get [MRV3, 2.10], that is the same conditions as in Theorem 8 but the omitted points are fixed. We can also obtain [V2, 19.2] that have the normality condition for a family of $K$-quasiconformal mappings. As corollaries to the last condition we can get [V2, 19.3-19.5].

REMARK. The following theorems are classical for conformal mappings and can be obtained by the result of [Z].

THEOREM 9. Let $\mathscr{F}=\{f\}$ be a family of conformal mappings $f: D \rightarrow \overline{\mathbf{C}} \backslash\{a, b\}$, $D \subset \mathbf{C}$. Then $\mathcal{F}$ is a normal family.

THEOREM 10. Let $\mathscr{F}=\{f\}$ be a family of conformal mappings $f: D \rightarrow \overline{\mathbf{C}} \backslash\left\{a_{f}, b_{f}\right\}$, $D \subset \mathbf{C}$ and $q\left(a_{f}, b_{f}\right) \geqslant \varepsilon>0, \forall f \in \mathscr{F}$, then $\mathscr{F}$ is a normal family.

\section{REFERENCES}

[MRV1] O. Martio, S. Rickman and J. Väisälä, Definitions for quasiregular mappings, Ann. Acad. Sci. Fenn. Ser. AI 448 (1969), 1-40.

[MRV2] _ Distortion and singularities of quasiregular mappings, Ann. Acad. Sci. Fenn. Ser. AI 465 (1970), 1-13. 
[MRV3] _ Topological and metric properties of quasiregular mappings, Ann. Acad. Sci. Fenn. Ser. AI 488 (1971), 1 - 31.

[R] S. Rickman, On the number of omitted values of entire quasiregular mappings, J. Analyse Math. 37 (1980), 100-117.

[Sa] J. Sarvas, Coefficients of injectivity for quasiregular mappings, Duke Math. J. 43 (1976), 147-158.

[SZ] S. Saks and A. Zygmund, Analytic functions, 3rd ed., Elsevier, New York, 1971.

[V1] J. Väisälä, On normal quasiconformal functions, Ann. Acad. Sci. Fenn. Ser. AI 266 (1959), 1-33.

[V2] _ Lectures on n-dimensional quasiconformal mappings, Lecture Notes in Math., vol. 229, Springer-Verlag, New York, 1971.

[Z] L. Zalcman, A heuristic principle in complex function theory, Amer. Math. Monthly 82 (1975), 813-817.

[Zo] V. A. Zoric, A theorem of M. A. Lavrentiev for space quasiconformal mappings, Math. USSR Sb. 74 (116) (1967), 417-433.

DEPARTMENT OF MATHEMATICS, UNIVERSITY OF KENTUCKY, LEXINGTON, KENTUCKY 40506

Current address: Department of Mathematics, Northern Illinois University, De Kalb, Illinois 60115 